\title{
Surgical Management of Intracranial Meningiomas in the Elderly: Early and Long-term Outcomes
}

This article was published in the following Dove Press journal:

Clinical Interventions in Aging

Tomasz Czernicki (D)

Department of Neurosurgery, Medical University of Warsaw, Warsaw, Poland
Purpose: In view of the risk associated with the surgical treatment of intracranial meningiomas in the elderly population due to the physiology of aging and multiple comorbidities, an attempt was made to identify factors influencing outcomes and to define the subgroup of patients who should not be operated on due to poor results.

Patients and Methods: A retrospective analysis of 58 patients over 70 years old with assessment of short-term and long-term outcomes. Scores by previously described CRGS, SKALE, and GSS grading systems were also calculated for our patients.

Results: Neurological morbidity was only associated with a critical location according to the SKALE grading system $(P=0.02)$. Six patients $(10.3 \%)$ died. Mortality was associated with the Karnofsky Performance Scale score (KPS $\leq 60$ vs KPS $\geq 70 ; P=0.0162$ ), the American Society of Anesthesiologists scale status (ASA 1 or 2 vs ASA $3 ; P=0.0022$ ) and the WHO grade of meningiomas $(P=0.012)$. Risk factors for tumor recurrence (six patients) were WHO grade $(P=0.00048)$ and Simpson grade of resection $(P=0.0437)$. At follow-up, excluding patients who died due to surgery or recurrence $(15.5 \%)$, most patients improved $(50 \%)$ or remained unchanged $(25.9 \%)$ in relation to the preoperative KPS status.

Conclusion: Postoperative neurological deterioration was only associated with a critical tumor location (skull base, eloquent area, large vessels involvement by the tumor). Due to a significantly higher risk of death, careful consideration should be taken for surgery in patients in a poor functional condition (KPS $\leq 60$ ) or in a poor physical condition (ASA 3 status). An improvement or at least nonworsening of the neurological status in relation to the preoperative condition was observed in the majority of patients during follow-up.

Keywords: intracranial meningioma, elderly, brain surgery, prognostic factors, complications, recurrence, survival

\section{Introduction}

The incidence rate of intracranial meningiomas increases progressively with age and it is a common disease in the elderly population, both in men and in women. ${ }^{1-4}$ The incidence in persons older than 70 years is 3.5 times higher than in younger people. ${ }^{3}$ With constantly increasing life expectancy and increasing use of diagnostic neuroimaging studies, an even higher number of detected cases of meningiomas in this age group of patients may be expected in the coming years. Treatment modalities include surgical resection, radiosurgery and watchful waiting. Surgery in elderly patients may pose more threats than in younger patients due to the physiology of aging and multiple comorbidities in these patients. ${ }^{1,5,6}$ The need to balance between the potential risks and benefits in the older age group challenges decision-making and the choice of management modality. Information on surgical
Correspondence: Tomasz Czernicki Department of Neurosurgery, Medical University of Warsaw, Banacha Street IA Warsaw 02-097, Poland

Tel +48225992575

Fax +48225991574

Email tczernicki@wp.pl 
outcomes is important for treatment planning, including the identification of patient subgroups who would benefit in the event of surgery selection in terms of overall survival and quality of life. Because decisions regarding the best therapeutic approach should be taken with the utmost care, ${ }^{7}$ I decided to present our experience in the surgical treatment of intracranial meningiomas in the elderly population aged over 70 years.

\section{Patients and Methods}

\section{Subjects}

The results of intracranial meningioma surgeries were retrospectively assessed in 58 patients from the age of 70, operated in years: 2010-2016. Surgeries were performed by many neurosurgeons. Patients with tumor recurrence, treated conservatively or referred for radiosurgery were excluded from the study. Indications for surgery in our study group included: symptomatic patients with evident signs and symptoms, constantly deteriorating patients with initially modest symptoms, evident tumor growth, the presence of peritumoral edema. The American Society of Anesthesiologists (ASA) scale was used to assess preoperative physical status. The Karnofsky Performance Status (KPS) scale ${ }^{8}$ was used to assess the preoperative, postoperative and follow-up functional status. The location, largest dimension and tumor volume were determined on the basis of preoperative magnetic resonance imaging (MRI) examinations. The volume of tumors was calculated according to the formula: $\mathrm{V}=4 / 3 \times \pi \times 1 / 2 \mathrm{~A} \times 1 / 2 \mathrm{~B} \times 1 / 2 \mathrm{C}$; where $\mathrm{A}, \mathrm{B}$ and $\mathrm{C}$ are the largest dimensions of the tumor in three perpendicular planes. Peritumoral edema was graded as severe if midline shift was present, as moderate if it was only perilesional, or as absent. Intraoperative neuronavigation and electrophysiological monitoring were used as adjuncts to microsurgical tumor resection in selected justified cases. The extent of tumor removal was classified according to the Simpson classification. ${ }^{9}$ Evaluation of the Simpson resection grade was assessed on the basis of the operating neurosurgeon's observation and was confirmed by postoperative computed tomography (CT) and MRI. A recurrence was defined as a reappearance of tumor in cases of complete meningioma removal or as proliferation of the tumor remnant in cases of incomplete meningioma removal in a series of followup MRI examinations. Most commonly, the first postoperative follow-up MRI examinations were performed half a year after the surgery and subsequent examinations were performed approximately yearly thereafter. MRI examinations were also performed in addition to the abovementioned scheme whenever neurological deficits appeared or worsened. Neurological evaluations were conducted at the time of admission, in the postoperative period, at the time of discharge, and every time during follow-up visits in our outpatient clinic. Perioperative mortality was defined as death within one month after surgery.

In addition, three proposed grading systems for surgical indication in the elderly patients with intracranial meningiomas, ie CRGS, SKALE and GSS, were calculated for each patient. The Clinical-Radiological Grading System (CRGS) is based on six criteria including the size and location of the tumor, neurological condition, KPS score, presence of peritumoral edema and presence of concomitant diseases. Among the subitems, size is referred as the largest dimension. The location of the meningioma is considered critical if it is close to major cerebral vessels, cranial nerves or the brainstem and eloquent areas, and subdivided into highly or moderately critical. Edema is defined as severe if associated with a shift of midline structures, moderate if only peritumoral or absent. Neurological deficits are referred as unrecoverable if stabilized and complete, otherwise as recoverable or absent. Concomitant diseases were defined as decompensated (uncontrolled), compensated or absent. A score above 10 (possible scoring range: 6-18) has been established as a cutoff for better outcomes and reflects postoperative three-month and one-year mortality. In patients who scored 10-12, the prognosis was positively affected by surgery and those with a score higher than 12 had a very good prognosis. ${ }^{10}$ The SKALE system includes sex (female sex as a positive prognostic factor), KPS score, ASA classification score, tumor location (considered critical if at the cranial base or in the eloquent area or near the large vessels) and peritumoral edema (graded as severe if the ratio of maximum radius of edema to the maximum diameter of the tumor was above 1; otherwise graded as moderate or absent). An increased one-year mortality was found if the SKALE score was less than eight, and no mortality if score was above eight (possible scoring range: $0-16) .{ }^{11}$ The Geriatric Scoring System (GSS) includes tumor size, neurological deficits (graded as progressive, stable severe and none or minor), KPS score, tumor location, peritumoral edema (graded as severe, mild and absent) and presence and severity of diabetes mellitus, hypertension or pulmonary disease. ${ }^{5}$ Improved outcomes 
were reported by authors creating GSS with scoring $>16$ points (possible scoring range: $8-24$ ).

\section{Statistical Analysis}

Data were analyzed using Statistica software, version 12.0 (Statsoft Inc., Tulsa, OK, USA). The analyses included basic descriptive statistics, the nonparametric the MannWhitney $U$-test and the Kruskal-Wallis test, as well as contingency tables with the Pearson chi-squared test and Fisher's exact test. A $P$-value of $<0.05$ was considered statistically significant.

\section{Ethics}

This study was retrospective and in our institution this type of research does not require Institutional Review Board approval. A statement was obtained from the IRB Committee (the Ethics Committee of the Medical University of Warsaw) that the study is exempt from such an approval (decision number: AKBE/220/2019). Because this study was conducted retrospectively from data obtained for clinical purposes and does not contain any personal patient data, the requirement that patients give informed consent was waived.

\section{Results}

\section{Study Group}

Our cohort of patients was aged between 70 and 85 years with a mean age of 75.1 years; slightly more than half of the patients were women $(55.2 \%)$. The mean duration of symptoms before the surgery was 9.2 months (range: five days to four years). The most common symptoms were motor deficits $(34.5 \%)$, seizures $(31 \%)$, headache $(24.1 \%)$, aphasia $(22.4 \%)$ and cerebellar symptoms $(8.6 \%)$. The tumors had a dural attachment: at the skull base in 24 patients $(41.4 \%)$, on the convexity in 22 patients $(37.9 \%)$ and in the parasagittal area or to the falx cerebri in 12 patients $(20.7 \%)$. The mean of the largest tumor dimension in our cohort was $45.6 \mathrm{~mm}$ (range: $19-89 \mathrm{~mm}$ ) and the mean tumor volume was $38.7 \mathrm{~cm}^{3}$ (range: $2.5-164 \mathrm{~cm}^{3}$ ).

Peritumoral edema was severe in $15.5 \%$, moderate in $58.6 \%$ and absent in $25.9 \%$ of the cases. Almost all patients, ie 53 patients, had comorbidities (with a median of 2 and with range from 0 to 9 comorbidities), as listed in Table 1. According to the ASA classification, 13.8\%, $70.7 \%, 15.5 \%$, and $0 \%$ of patients had ASA grades 1 to 4 , respectively. Patients status according the KPS scale is presented in Table $2 ; 12$ patients $(20.6 \%)$ were dependent
Table I Type and Frequency of Comorbidities

\begin{tabular}{|l|l|}
\hline Comorbidities & $\begin{array}{l}\text { Number of Patients } \\
\text { n (\%) }\end{array}$ \\
\hline Hypertension & $46(79.3)$ \\
Thyroid disease & $17(29.3)$ \\
Ischemic heart disease & $15(25.9)$ \\
Diabetes & $13(22.4)$ \\
Arrhythmia & $8(13.8)$ \\
Chronic obstructive pulmonary & $6(10.3)$ \\
disease & \\
Chronic heart failure & $3(5.2)$ \\
Chronic renal failure & $3(5.2)$ \\
Artificial heart valves & $2(3.45)$ \\
Liver disease & $1(1.7)$ \\
\hline
\end{tabular}

Table 2 Preoperative Functional Status According to the KPS Scale

\begin{tabular}{|l|l|}
\hline Preoperative KPS Status & Number of Patients n (\%) \\
\hline 40 & $1(1.7)$ \\
50 & $5(8.6)$ \\
60 & $6(10.3)$ \\
70 & $4(6.9)$ \\
80 & $11(19)$ \\
90 & $27(46.6)$ \\
100 & $4(6.9)$ \\
\hline
\end{tabular}

(KPS $\leq 60)$ and 46 patients $(79.4 \%)$ were independent (KPS $\geq 70$ ). The median CRGS score was 13 (range: 9-17), with $1.7 \%$ patients with a score $<10,39.7 \%$ with a score $10-12$ and $58.6 \%$ with a score $>12$. The median SKALE score was 12 (range: 6-14), with 8.6\% patients with a score $<8,12.1 \%$ with a score equal to 8 and $79.3 \%$ with a score $>8$; the median GSS score was 19 (range: $13-23$ ), with $86.2 \%$ of patients with a score $>16$.

\section{Surgical Procedures}

All operations were performed electively. Simpson grades I or II of resection were achieved in $81 \%$ of cases, Simpson grade III in $5.2 \%$ and Simpson IV in $13.8 \%$ of the procedures. More than half of the patients $(56.9 \%)$ had WHO grade I meningiomas, but $37.9 \%$ of patients had WHO grade II meningiomas and $5.2 \%$ of patients had WHO grade III meningiomas. Factors with an impact on Simpson grade of resection (I+II vs III vs IV) included location according to dural attachment $(P=0.0027)$, critical location of meningiomas according to definition in the CRGS and GSS grading systems $(P=0.003$ and $P=0.027)$ and total CRGS score $(P=0.0213)$. Other factors had no impact, including KPS 
status $(P=0.12)$, ASA status $(P=0.46)$, the presence and severity of peritumoral edema $(P=0.69)$, tumor volume $(P=0.47)$ and the largest dimension of the meningioma $(P=0.71)$. Illustrative cases are demonstrated in Figures $1-3$.

\section{Morbidity}

Before surgery, 40 out of 58 patients had neurological deficits, which deteriorated postoperatively in seven cases. In addition, new neurological deficits appeared postoperatively in five patients, giving a total of 12 cases $(20.7 \%)$ of neurological deterioration. However, among the 40 patients with preoperative neurological deficits, an improvement was observed in 14 patients (ie in 35\% of patients with preoperative deficits), including improvement to a normal neurological status in six patients $(15 \%)$. Internal complications occurred in 12 patients
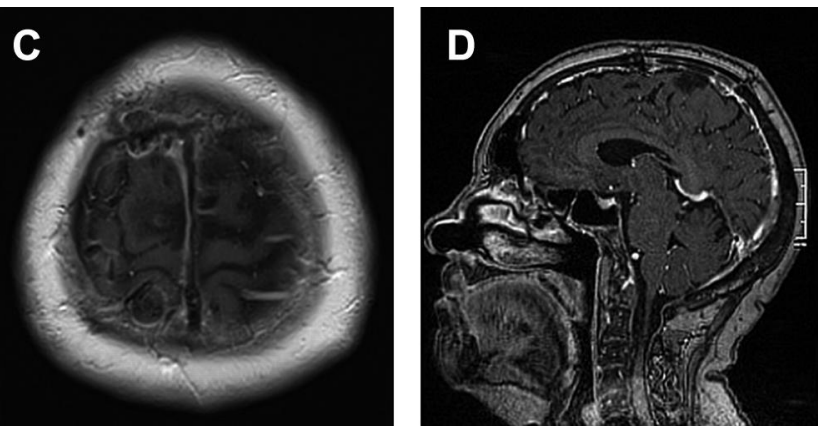

Figure I Preoperative contrast-enhanced TI-weighted MR images in the axial (A) and coronal (B) planes of a 7I-year-old woman operated on for a parasagittal meningioma. Last follow-up MRI examination obtained after Simpson grade III resection of a fibroblastic meningioma (WHO grade I) showed no recurrence in contrastenhanced TI-weighted images in the axial (C) and sagittal (D) planes.
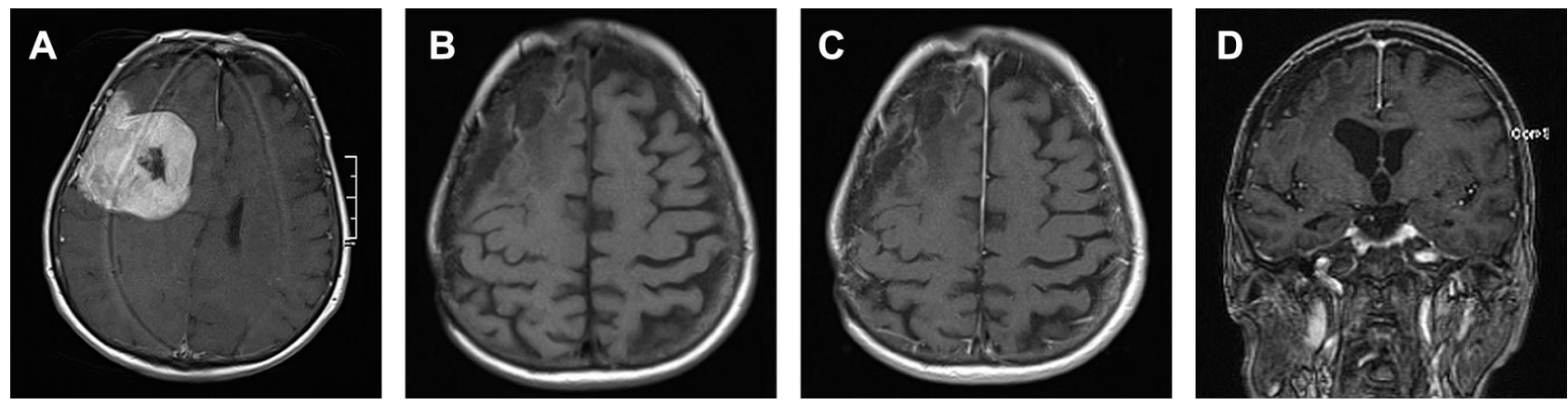

Figure 2 Preoperative contrast-enhanced TI-weighted MR image in the axial plane (A) of an 82-year-old woman operated on for a convexity meningioma(, , ). Last followup MRI examination obtained after Simpson grade I resection of an atypical meningioma (WHO grade II) with no postoperative radiotherapy showed no recurrence in the native TI-weighted image in the axial plane (B) and in contrast-enhanced TI-weighted images in the axial (C) and coronal (D) planes.
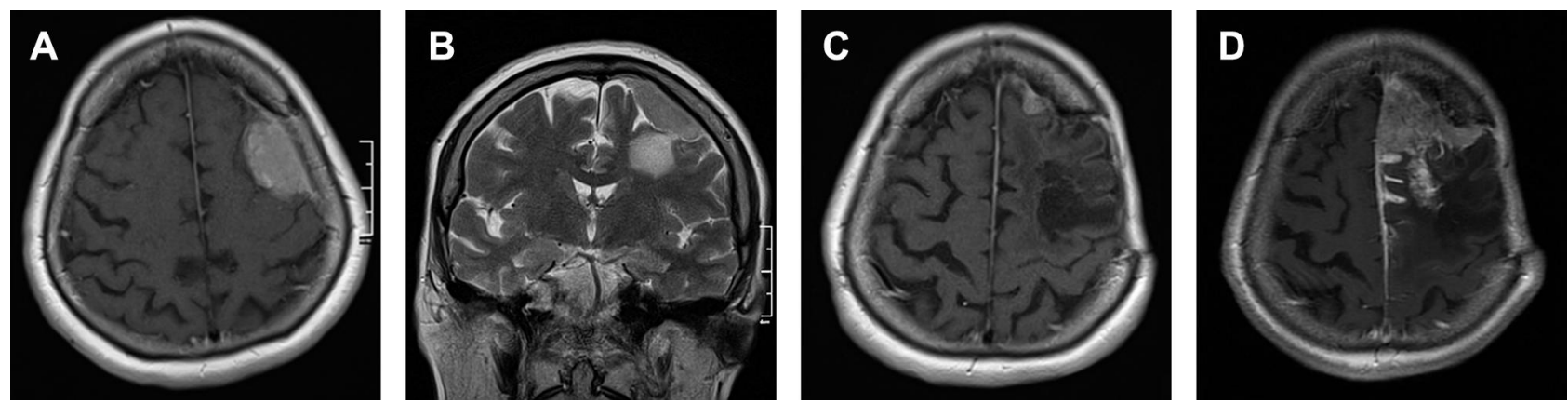

Figure 3 Preoperative MRI examination in contrast-enhanced TI-weighted axial $(\mathbf{A})$ and T2-weighted coronal (B) planes of a convexity meningioma in a 72 -year-old woman with Simpson grade I resection of an atypical meningioma (WHO grade II). No postoperative radiotherapy was used. MRI examination performed I8 months after surgery revealed a small recurrence shown in the axial contrast-enhanced TI-weighted image (C). Cyberknife radiotherapy was used. MRI examination performed 42 months after surgery showed further tumor growth (D) and Cyberknife radiotherapy was reapplied. The patient was alive at the last follow-up contact ( 50 months post-surgery). 
(20.7\%), including pulmonary in six cases and cardiovascular in two cases. Overall morbidity defined as postoperative neurological deterioration and/or important internal complication occurred in 18 patients $(31 \%)$.

Neurological morbidity was not related to preoperative KPS score $(P=0.11)$, ASA status $(P=0.56)$, location of dural attachment of the meningiomas $(P=0.58)$, presence and severity of peritumoral edema $(P=0.5)$, the largest tumor dimension $(P=0.36)$, tumor volume $(P=0.766)$, extent of removal according the Simpson classification ( $P=0.328$, Table 3$)$ and WHO grade $(P=0.65)$. A critical location of the meningiomas according the CRGS and SKALE grading systems had an impact on increasing the incidence of postoperative neurological deterioration, with $P=0.06$ (statistical trend) and $P=0.02$, respectively.

Overall morbidity was not related to preoperative KPS score $(P=0.586)$, location of the dural attachment of the meningiomas $(P=0.89)$, presence and severity of peritumoral edema $(P=0.91)$, the largest tumor dimension $(P=0.786)$, tumor volume $(P=0.414)$, extent of removal according the Simpson classification $(P=0.274$, Table 3$)$ and WHO grade $(P=0.162)$. A higher overall morbidity (66.7\%) was found in patients with ASA Class 3 status in relation to patients with ASA Class 1 or 2 status (24.5\%) $(P=0.034)$. Moreover, a critical location of the meningiomas according to the SKALE grading system showed a statistical trend to higher morbidity $(P=0.0545)$.

\section{Mortality}

Six patients $(10.3 \%)$ died in the postoperative period. The mortality rate was higher in men $(23.1 \%)$ than in women $(0 \%), P=0.015$. The cause of death was directly linked to surgery in three cases and, in the remaining three patients, death was associated with other postoperative complications. The reasons for deaths in individual cases were as follows: massive venous stroke resulting from venous thrombosis following surgery, ischemic stroke of the brainstem due to occlusion of tumor-encased arteries during surgery, meningitis, urosepsis, pneumonia, and myocardial infarction.
Postoperative mortality was lower in patients with a preoperative KPS score $\geq 70(4.35 \%)$ compared to those with a KPS score of 60 and below (33.3\%), $P=0.0162$, and was lower in patients with ASA Class 1 or 2 status (4.1\%) compared to those with ASA Class 3 status (44.4\%), $P=0.0022$. The location of the meningiomas $(P=0.49)$ and the presence and severity of peritumoral edema $(P=0.86)$ were not be associated with mortality. Likewise, there was no relationship between mortality and maximal tumor dimension $(P=0.312)$, tumor volume $(P=0.35)$, or with the extent of tumor removal according to the Simpson classification, $P=0.113$; Table 3 . No mortality was observed in WHO grade I meningiomas, in contrast to WHO grade II and III meningiomas, with a mortality rate of $24 \%$, $P=0.012$.

None of the comorbidities ( $P=\mathrm{ns}$ ) had an impact on the incidence rates of neurological morbidity, overall morbidity, and mortality. The patients with postoperative complications, ie neurological morbidity or overall morbidity or those who died in the postoperative period were not found to have on average more comorbidities than the other patients $(P=0.476, P=0.266$, and $P=0.91$, respectively).

The scores by the grading systems, ie CRGS, SKALE and GSS, had no impact on the neurological morbidity, a variable impact on the overall morbidity, and a significant impact on mortality (Table 4).

\section{Follow-up and Recurrences}

Fifty out of 52 surviving patients were followed-up for a median period of 48.25 months (mean: 75.6 months; range: 5.5-110 months). Two patients were lost to follow-up.

There were six cases (12\%) of recurrence, after 3, 18, 23, 41, 88 and 97 months, respectively. Among them, four patients had histological WHO grade II and 2 had WHO grade III meningiomas. Among the four patients with WHO grade II meningiomas: in two patients with partial excision (Simpson IV) postoperative radiotherapy was performed and in two patients with complete tumor removal (Simpson I) no radiotherapy was planned. In both patients with WHO grade III tumors, postoperative radiotherapy was planned, but one of them refused further treatment. The course of the disease

Table 3 Neurological Morbidity, Overall Morbidity and Mortality in Relation to the Simpson Grade of Removal

\begin{tabular}{|l|l|l|l|}
\hline Extent of Removal (Simpson Grade) & Neurological Morbidity n (\%) & Overall Morbidity $\mathbf{n}(\%)$ & Mortality $\mathbf{n}(\%)$ \\
\hline I + II $n=47$ & $9(19.15)$ & I3 (27.7) & $3(6.4)$ \\
III $n=3$ & 0 & I (33.3) & I (33.3) \\
IV $n=8$ & $3(37.5)$ & $4(50)$ & $2(25)$ \\
\hline
\end{tabular}


Table 4 The Relationship Between the Grading Scales' Scores and the Incidence of Neurological Morbidity, Overall Morbidity, and Mortality

\begin{tabular}{|l|l|l|l|}
\hline Grading Scale & $\begin{array}{l}\text { Neurological } \\
\text { Morbidity }\end{array}$ & $\begin{array}{l}\text { Overall } \\
\text { Morbidity }\end{array}$ & Mortality \\
\hline CRGS score & $P=0.108$ & $P=0.017 I^{\mathrm{a}}$ & $P=0.017^{\mathrm{a}}$ \\
SKALE score & $P=0.56$ & $P=0.067$ & $P<0.005^{\mathrm{a}}$ \\
GSS score & $P=0.35$ & $P=0.152$ & $P=0.0494^{\mathrm{a}}$ \\
\hline
\end{tabular}

Note: ${ }^{\text {a}}$ Significant relationships.

in patients with tumor recurrences is presented in Table 5. Three patients $(6 \%)$ died due to tumor recurrences and 10 other patients $(20 \%)$ died during follow-up for other reasons.

The main risk factor for tumor recurrence was the WHO grade of the meningiomas; in WHO grades I, II, and III, risk of recurrence was $0 \%, 25 \%$ and $66.7 \%$, respectively $(P=0.00048)$. In addition, recurrence risk was associated with the extent of resection, and was in Simpson grades I and II: 7.1\%, Simpson grade III: $50 \%$ and Simpson grade IV: $33.3 \%(P=0.0437)$. The location of dural attachment and tumor size (largest dimension and volume) were not related to the recurrence rate $(P=0.38$, $P=0.51$, and $P=0.51$, respectively).

At follow-up (before eventual death due to other reasons), it was found that nine patients $(15.5 \%)$ died (six in the postoperative period and three from recurrences), and among other patients, except two cases (3.4\%) who were lost to follow-up, 29 (50\%) improved, 15 (25.9\%) remained unchanged and three $(5.2 \%)$ deteriorated in relation to the preoperative status according to the KPS scale. It is worth noting that, among the abovementioned 15 patients whose condition did not change, in four $(6.9 \%)$ patients the condition could not improve, because their preoperative and follow-up status was $100 \mathrm{KPS}$. The preoperative KPS of 47 patients (excluding deceased patients due to surgery or recurrence) improved at last follow-up from a mean score of $82.6 \pm 13.6$ to $93.4 \pm 10.7$ (median from 90 to $100 \mathrm{KPS}$ ).

\section{Discussion}

An increase in the average length of life and the more frequent use of diagnostic neuroimaging studies in recent years have resulted in an increasing number of detected meningioma cases, especially in the elderly population. Every day, neurosurgeons are faced with the dilemma of whether to operate on meningiomas in patients in this age group. ${ }^{5,12-18}$ Surgery in the elderly population may be associated with a risk of serious or even life-threatening postoperative complications due to the physiology of ageing and multiple comorbidities. ${ }^{1,5,6,10,11,13}$ However, it should also be noted that the intraoperative and postoperative management of intracranial lesions improved dramatically in recent decades and surgical treatment of intracranial meningiomas has become a valid therapeutic option as well in the elderly population. The reported short-term mortality rates range from $0 \%$ to $13.5 \% \%^{1,4-6,10-16,19,20}$ and overall complication rates range between $3 \%$ and $30 \%$. $^{1,4-6,11-13,16,19,20}$ It is worth noting that is difficult to make direct comparisons regarding morbidity between studies because the definitions of complications are rarely described in detail. In our cohort, the mortality rate was $10.3 \%$, the neurological morbidity rate was $20.7 \%$ and the overall morbidity rate, including neurological deterioration as well as severe, life-threatening, internal complications was $31 \%$.

Table 5 The Course of Patients with Tumor Recurrence

\begin{tabular}{|l|l|l|l|l|l|l|}
\hline Case & $\begin{array}{l}\text { WHO } \\
\text { Grade }\end{array}$ & $\begin{array}{l}\text { Simpson } \\
\text { Grade }\end{array}$ & Postop Rth & $\begin{array}{l}\text { Time to } \\
\text { Recurrence }\end{array}$ & $\begin{array}{l}\text { Additional } \\
\text { Treatment }\end{array}$ & $\begin{array}{l}\text { Last Patient Status (Length } \\
\text { of Follow-up) }\end{array}$ \\
\hline 78 -year-old man & II & IV & Yes & 4 I months & $\begin{array}{l}\text { Reoperation, GKS, } \\
\text { reoperation }\end{array}$ & Deceased (75 months) \\
\hline 74 -year-old man & II & IV & Yes & 97 months & $\begin{array}{l}\text { No; watchful } \\
\text { observation }\end{array}$ & Alive (II0 months) \\
\hline 74 -year-old woman & II & I & No & 88 months & $\begin{array}{l}\text { No; watchful } \\
\text { observation }\end{array}$ & Alive (I06 months) \\
\hline 72 -year-old woman & II & I & No & 18 months & $2 \times$ Cyberknife & Alive (50 months) \\
\hline 76 -year-old man & III & I & Yes & 23 months & Chemotherapy & Deceased (26 months) \\
\hline 85 -year-old man & III & III & Refused & 3 months & No & Deceased (5.5 months) \\
\hline
\end{tabular}


The extent of meningioma resection in the elderly population is similar to younger counterparts, ${ }^{21-23}$ with complete resection being achieved in most cases (in about $72-89 \%$ ). ${ }^{12-15,19,20,22,23}$ Our percentage of resection was comparable with these results. The extent of tumor resection is very important because the more radical the surgery, the smaller the risk of recurrence. ${ }^{12,18,21,23,24}$ Complete removal is most often possible in convexity meningiomas, followed by parasagittal and falcine meningiomas, while it is least possible with skull base meningiomas, ${ }^{14,20,25}$ which is consistent with our results. The worst results with regard to the radicality of the operations in the case of skull base meningiomas are associated with involvement of vessels and cranial nerves, tumors adhesion to the brain stem, cavernous sinus involvement and the presence of blind points during the surgical approaches.

Many investigators have attempted to define preoperative indicators of morbidity in the elderly population, with several authors demonstrating the predictive value of more advanced patient age, ${ }^{1,6}$ male sex, ${ }^{1,10,11}$ tumor size,,${ }^{11,14}$ tumor location, ${ }^{6,11}$ presence and severity of edema, ${ }^{11,14}$ and general health indicators, such as ASA and KPS scores, ${ }^{11,15}$ whereas some authors have negated the value of these parameters. ${ }^{17-20}$ Conflicting results and divergent prognostic factors highlight the importance of future detailed investigations in morbidity analysis. In our cohort, it confirmed an often described fact that patients in poor physical status (ASA class III) fared worse than patients in good health condition (ASA class I or II). ${ }^{11,15}$ Unlike some others, ${ }^{11,15}$ in the present study, there was no significant impact of worse preoperative functional status according to the KPS scale on postoperative morbidity. ${ }^{20}$ It seems reasonable to assume that patients with many comorbidities may have a worse postoperative course, but were no correlations between the number of comorbidities and the complication rate. Furthermore, there were no specific comorbidities in the data forcing us to refuse surgery.

In our material, it was found that the risk of death after surgery was affected by worse preoperative status (KPS $\leq 60$ vs KPS $\geq 70$; ASA 3 vs ASA 1 or 2 ), male sex and tumor malignancy (WHO grade II or III vs WHO grade I). Dobran et $\mathrm{al}^{15}$ also found higher mortality in patients with ASA class 3 status compared to patients with ASA class 2 status, but did not find a correlation between the preoperative KPS status or gender and one-month mortality. In turn, Sacko et $\mathrm{al}^{11}$ found no postoperative mortality, but the one-year mortality was lower in women, in patients with the KPS score of 60 or greater, in patients with ASA class of 1 or 2 , in those with a noncritical tumor location and in cases with moderate or absent peritumoral edema. In a series of 37 patients operated on in the ninth decade of life, D'Andrea et al ${ }^{14}$ stated a significant impact of worse physical status (ASA class 3) and worse functional status $($ KPS $\leq 60)$ on higher mortality.

It seems that the accurate preoperative selection of patients who can benefit from surgery will be fundamental to achieving better results in the future. For that purpose, different studies have proposed several scoring systems to facilitate the decision-making process. Striving to develop surgery indication scoring systems incorporating several probable prognostic factors seems more justified than relying on single factors. Caroli et $\mathrm{al}^{10}$ found that higher scores in their Clinical-Radiological Grading System and female sex significantly correlated with lower three-month and one-year mortality. Among the subset items of CRGS, the following had no impact on mortality: size and location of the tumor, preoperative KPS score and preoperative neurological status; however, two factors showed a trend towards statistical significance: the presence of edema increased three-month mortality and the presence of concomitant diseases increased one-year mortality. Sacko et $\mathrm{al}^{11}$ created the SKALE grading system in which patients with a score greater than eight had an excellent outcome, whereas those with a SKALE score of less than eight had a poor outcome. All subset items of SKALE decreased postoperative mortality in their material in the following manner: female sex, KPS score $\geq 60$, ASA Class 1 or 2 , a noncritical tumor location and moderate or absent peritumoral edema. Larger tumor size, critical location, severe peritumoral edema and total surgical excision were associated with a higher risk of postoperative complications in their cohort. Cohen-Inbar et $\mathrm{al}^{5}$ constructed the Geriatric Scoring System (GSS) in which patients may score between 8 and 24; higher scores are associated with a better outcome, including lower mortality rate, lower one-year recurrence rate and better five-year functional outcome (KPS, Barthel Index, and Glasgow Coma Scale). According to their calculations, patients presenting with a score equal to or greater than 15 may benefit from surgical intervention, in contrast to patients with lower scores. The same authors validated the GSS scale one year later based on a retrospectively assessed group of patients, showing better outcomes in patients with a score greater than 16 points. Grossman et $\mathrm{al}^{1}$ used the Charlson Comorbidity Score (CCS) and found it to be 
positively correlated with complications and mortality. In their study, increasing age was correlated with the rate of neurological deteriorations and respiratory complications, but not with cardiac complications and strokes. These associations highlight the importance of comorbidities on surgical outcomes in the elderly population.

The value of the abovementioned grading systems as predictors of postoperative morbidity and mortality risk has not been unanimously confirmed in the literature, ${ }^{19,20,26,27}$ with some authors reporting no relationship or a relationship of only some of them with the outcome. ${ }^{19,20}$ Delgado-Fernández et $\mathrm{al}^{26}$ confirmed a predictive value of CRGS, SKALE and GSS on morbidity and mortality, in turn Konglund et $\mathrm{al}^{19}$ reported that none of the grading systems had any effect on morbidity and only the SKALE score was associated with mortality, while other grading systems, ie CRGS, GSS and CCS, had also no impact on mortality.

The risks of surgical removal must be balanced against the availability of other treatment modalities such as stereotactic radiosurgery. ${ }^{2,25,28,29}$ It is often difficult to determine the optimal management strategy in an individual patient. Oligosymptomatic, in particular, small meningiomas may be treated with stereotactic radiosurgery (SRS) to achieve a long-term tumor control rate comparable to surgery results. ${ }^{2,25,28,29}$ SRS is associated with small, acceptable morbidity and is especially suitable for skull-base meningiomas that are less accessible for surgery. ${ }^{25,28,29}$ For large-sized meningiomas, SRS is not feasible, and moreover surgical resection relieves the mass effect immediately and alleviates or causes the withdrawal of symptoms and signs. ${ }^{25}$ Local tumor control after radiosurgery is clearly higher for WHO grade I meningiomas compared to WHO grade II and WHO grade III meningiomas. ${ }^{2,25,29}$ Radiosurgery may also lead to complications in the form of adverse radiation effects or neurological deterioration. ${ }^{25,28}$ It should be remembered that meningiomas are usually benign tumors and thus, whenever it is possible without a significant risk, surgical removal should be preferred. Surgery also provides a certain histopathological diagnosis, which is also an important advantage.

Some authors consider meningiomas in elderly patients as a separate clinical entity characterized by slow progression and reduced aggressive behavior owing to different cellular proliferation, vascularity and intratumoral hormonal profiles. The reported average growth rate of benign intracranial meningiomas is a few millimeters per year or over a dozen percent of tumor mass per year. ${ }^{30,31}$ In all age groups, an inverse correlation is found between calcifications and tumor growth rate, with even no tumor growth at all in cases with progressive calcifications. ${ }^{30,32}$ It has also been described a dependence that if tumor size increases with time, its further growth rate decreases. ${ }^{32}$ Understanding the meningioma growth pattern in the elderly population has profound implications for tumors management. Meningiomas discovered in the elderly may have a more indolent course than those discovered in younger patients. $^{30,31}$ There are alternatives to subjecting older patients to the risk of surgery and anesthesia. Some patients with slow growing meningiomas may be observed for clinical changes or may be treated nonsurgically with radiosurgery. Radiosurgery, constituting an additional and alternative treatment for these patients complicates the decision-making process of qualification for surgery even more. Moreover, considering the advancements in surgical techniques and anesthetic care, the rate of successful surgical treatment has dramatically increased in recent decades. Some authors suggest that the best surgical approach should be to remove as much tumor as possible, but not necessarily completely, in order to minimize the risk of new neurological deficits. ${ }^{11}$

The one-year recurrence rate of meningiomas ranges from $5.4 \%$ to $11.5 \%,{ }^{4,11}$ whilst the five-year recurrence rate ranges from $8 \%$ to $24.1 \%{ }^{2,4,12}$ In our cohort, a recurrence was observed in $12 \%$ of patients, with the one-year recurrence rate of $2 \%$ and with five-year recurrence rate of $8 \%$. Histopathological WHO grade of meningiomas ${ }^{2,15,21}$ and the extent of their resection ${ }^{2,12,18,21,23,24}$ were identified as the main risk factors for tumor recurrence in this study. Previous studies have made similar observations, ${ }^{2,12,15,18,21}$ although this has not been a universal finding. ${ }^{14}$ Regarding a more radical extent of the resection, the risk of neurologic morbidity must be weighed against the potential benefits of tumor removal completeness, ${ }^{12}$ but in our cohort there were no associations between the radicality of tumor resection and the risk of neurological deterioration. Bir et $\mathrm{al}^{12}$ in their series of benign meningiomas (WHO GI), in addition to the extent of resection according Simpson classification, found many other predictors for recurrence such as location at the skull base, larger tumor size, worse preoperative functional status according to KPS, the presence and number of comorbidities and GSS score below 16. If surgery is performed without complete tumor removal, a decision must be made postoperatively whether to follow the patient with serial MRI or to proceed directly to some form of radiation therapy. ${ }^{33}$ It is known that incompletely removed benign meningiomas can 
also recur, most often in the period from 5 to 15 years after surgery. ${ }^{21}$ Postoperative radiotherapy in atypical meningiomas is justified and in anaplastic meningiomas is obligatory due to a high risk of recurrence. ${ }^{2,24}$ Patients with diffuse tumor remnants are typically the best candidates for external beam radiation therapy, whereas patients with tumor remnants that are nodular or close to vital structures can be better managed with stereotactic radiosurgery. ${ }^{25}$

All recurrences in our material were only observed in atypical and anaplastic meningiomas, with the tumor relapse rate as high as $25 \%$ and $66.7 \%$, respectively. ${ }^{2,24,33}$ While the need for radiotherapy use immediately after anaplastic meningioma surgery or nontotal excision of atypical meningioma does not raise any doubts, ${ }^{2,24,34,35}$ a legitimate question can be asked whether not to apply obligatory radiotherapy also directly after the total removal of an atypical meningioma instead of watchful observation and applying radiotherapy at the time of recurrence (with or without reoperation). ${ }^{33}$ However, the role of direct postoperative radiotherapy for totally removed atypical meningiomas is still not completely explained, ${ }^{2}$ with some authors $^{24,34,35}$ not finding a significant reduction of the recurrence rate in patients treated with radiotherapy compared to those not subjected to radiotherapy after surgery. Lee et $\mathrm{al}^{24}$ recommended in such cases postoperative radiotherapy only in a subgroup of patients older than 55 years with mitotic activity and sheeting in the histopathological examination. Mair et $\mathrm{al}^{34}$ suggested that radiotherapy is not justified in cases with Simpson grade 1 or 2 of resection of atypical meningiomas, but patients with nontotal resection should be treated with radiosurgery, while postoperative fractionated irradiation should be reserved for tumor remnants too large for radiosurgery. Until now, no effective chemotherapy has been developed for the treatment of atypical and anaplastic meningiomas. ${ }^{2}$

The results comparing mortality in elderly patients to younger patients are conflicting, with some authors 6,7 reporting greater mortality in the older population in opposition to results of other investigators reporting similar mortality. ${ }^{13,16,17,20,22}$ Likewise, the results regarding the incidence rates of postoperative neurological deterioration between older and younger patients assessed in long-term follow-up are inconsistent. ${ }^{13,17,20}$ And finally, analyses focusing on the overall complication rate also yielded conflicting data with some investigators, ${ }^{6,13,16,22}$ but not all, ${ }^{17,20}$ showing that elderly patients have a higher overall complication rate. However, when looking at their structure, elderly patients have minor complications more frequently and have a similar number of serious complica-

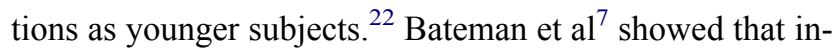
hospital mortality, adverse outcomes, and the length of hospital stay were all significantly higher after meningioma resection in the elderly population and revealed a sharp increase in the mortality rate among patients aged 70 and older. The mortality rate for patients aged 50 to 59 years and those aged 60 to 69 years was $1.4 \%$ and $1.2 \%$, respectively, while the rate for patients aged 70 to 79 years was $3 \%$ and for patients aged 80 and older was $7 \%$. Similarly, the rate of adverse outcomes in their cohort increased with each decade of life, reaching a high of $48 \%$ for patients aged 70 to 79 years and $67.9 \%$ in those aged 80 and older. The length of hospital stay also demonstrated a large effect of age. These authors also found that the increase in the risk of mortality, adverse outcomes and the length of hospital stay for meningioma resection mirrors those seen for other major surgical procedures (ie hemicolectomy, sigmoidectomy, open cholecystectomy) performed in the elderly. In contrast, Slot et $\mathrm{al}^{17}$ showed no significant difference in mortality, complication rates and the length of hospital stay between older and younger patients despite the fact that the elderly group ( $\geq 65$ years old) was characterized by a worse physical status according to the ASA scale and a worse functional status according to KPS. The same results were obtained when they set the cutoff age for the elderly population at 70 years. The only difference found in their study was a worse neurological status of older patients at 6-12 months after discharge, but this disappeared at the time of the next followup, ie at 12-18 months after the discharge. Boviatis et al, ${ }^{13}$ comparing treatment results between 108 patients aged $\geq 65$ years old and 240 younger patients operated on for supratentorial meningiomas, found no difference in mortality; however, neurological deterioration and complications were more frequently observed in the older patients. Interestingly, the incidence of cardiorespiratory complications was similar in both patient groups in that study. In summary, due to the fact that nowadays older people remain active and independent for a longer time, many neurosurgeons recommend a surgical approach for meningiomas also in the elderly population, despite a greater possibility of morbidity and perhaps of mortality. ${ }^{15}$ Furthermore, advances in perioperative care and improving the overall health status of the elderly patients are conducive to making decisions regarding surgery.

Advances in medical care and surgical technique prompt important questions about the indications for 
surgery and treatment results in the geriatric population not only after surgery for intracranial meningiomas, but also for other tumors occurring in the central nervous system. The most important prognostic factors highlighted in many reports related to glioma surgery in the elderly patients include increasing age, ${ }^{36,37}$ the extent of resection, ${ }^{36-38}$ cognitive function, ${ }^{37}$ functional status, ${ }^{37,38}$ nutritional status, ${ }^{37}$ and the presence and severity of comorbidities. ${ }^{37}$ The overall and progression-free survival times are significantly longer in cases of complete tumor resection compared to partial resection or biopsy alone. $^{36-38}$ O-6-methylguanine-DNA methyltransferase (MGMT) promoter methylation status ${ }^{36-38}$ and isocitrate dehydrogenase (IDH) gene mutation ${ }^{37,38}$ are also important prognostic factors, as with nonelderly patients. In the group of 322 patients above 65 years of age operated on for intracranial glioblastoma, Ius et $\mathrm{al}^{36}$ found the one-year overall survival and progression-free survival rates of $42.07 \%$ and $24.8 \%$, respectively. Younger age, higher extent of resection, no corpus callosum involvement, lower T1/T2 MRI index, and the presence of MGMT promoter methylation were independent survival risk factors in their cohort. According to results used by them, classification and regression tree (CART) method, surgery may be considered the first therapeutic option if preoperatively estimated extent of resection is greater than $80 \%$. According to the current evidence, postoperative radiotherapy and chemotherapy are of benefit and have been relatively well tolerated in the elderly glioma cohort. ${ }^{37,38}$ Satisfactory treatment results in the elderly patients undergoing intracranial procedures have also been observed after surgery for benign tumors, such as pituitary adenomas and vestibular schwannomas. ${ }^{39-42}$ Tardivo et $\mathrm{al}^{40}$ reported only two postoperative deaths in their cohort of 81 elderly patients who underwent transsphenoidal surgery for pituitary adenomas, with a low rate of postoperative complications (cerebrospinal fluid rhinorrhea in four, diabetes insipidus in three, hypopituitarism in 15) and a high rate of visual improvement (in 20 of 35 patients with a preoperative visual deficit). In turn, Zhao et $\mathrm{al}^{42}$ in their series of 130 elderly patients operated for pituitary adenomas reported 12 cases of postoperative hypopituitarism and 11 cases with other complications, including two severe ones. Zhan et $\mathrm{al}^{41}$ showed that the rate of complications after endoscopic endonasal transsphenoidal approach for nonfunctioning pituitary adenomas did not differ between 158 elderly patients aged above 65 years compared to 155 younger patients. Likewise, Bowers et $\mathrm{al}^{39}$ found similar rates of complications and similar rates of facial nerve outcome and hearing preservation in the groups of elderly and younger patients, concluding that age alone may not be a contraindication for vestibular schwannoma surgery. However, Sylvester et $\mathrm{al}^{43}$ in their study based on the national inpatient sample arrived at an opposite conclusion as they found higher in-hospital overall complication and mortality rates in older patients operated on for vestibular schwannomas, while emphasizing that although mortality was higher in the elderly, deaths were actually rare.

The percentages of atypical and anaplastic meningiomas in our material was high at $37.9 \%$ and $5.2 \%$, respectively. This may be associated with the fact that indications for surgery in our cohort were, among others, evident rapid tumor growth as well as the presence of peritumoral edema, both factors frequently associated with atypia or malignancy in meningiomas. Yamamoto et $\mathrm{al}^{20}$ demonstrated that intracranial meningiomas in the elderly population had a significantly higher proliferation index (Ki-67) compared to meningiomas in younger counterparts. It is also very important that the latest edition of the WHO classification of tumors of the central nervous system has changed the grading of meningiomas using a combination of criteria such as an increased mitotic index, the presence of brain invasion, and histological tumor features including loss of lobular architecture, hypercellularity, presence of small cells with high nuclear to cytoplasm ratio, presence of large and prominent nucleoli and presence of foci of spontaneous necrosis. These changes have resulted in a higher percentage of intracranial meningiomas being classified as WHO grade II, ie atypical meningiomas. ${ }^{33,35}$ In older reports, less than $5 \%$ of meningiomas were classified as atypical, whereas nowadays up to $35 \%$ of meningiomas could be atypical. ${ }^{2,24,35}$ The percentage of patients with anaplastic meningiomas (WHO grade III) has remained relatively stable. ${ }^{2}$ Dobran et $\mathrm{al}^{15}$ also found $32 \%$ cases of atypical meningiomas in their cohort of 25 patients aged above 80 years operated on for intracranial meningiomas. Pearson et $\mathrm{al}^{35}$ retrospectively reviewed 471 meningiomas treated in their institution and noted that the incidence of atypical meningiomas was $4.4 \%$ among cases before 2000; after this, the recognition of atypical meningiomas increased systematically, reaching $35.5 \%$.

In the majority of patients who successfully survived the postoperative period, an improvement or at least a nonworsening neurological condition is observed during the long-term follow-up. ${ }^{14,15}$ Thus, patient outcomes 
limited to the status at discharge may underestimate the true mortality rate of meningioma resection and overestimate the long-term morbidity of the surgery. ${ }^{17}$ In our cohort, there was no mortality within a few months after surgery associated with postoperative complications, and an improvement in the functional condition was observed in half of surviving patients during long-term follow-up, with deterioration in a minority of patients.

The major limitation of this study is its retrospective nature, patients who were already selected for surgery with the same surgical policies, but however, without a precise protocol were assessed, thus making selection to some extent dependent on surgeons' experience and subjective assessments. In addition, the lack of an established protocol according to which patients with severe comorbidities should not be qualified for surgery may have also influenced the results. The fact that the surgeries were performed by many neurosurgeons with different expertise could also affect outcomes.

\section{Conclusions}

In most elderly patients, intracranial meningiomas can be completely removed with a morbidity and mortality risk within acceptable limits. The location of the tumor has an impact on the extent of resection, as tumors were completely removed in the majority of patients with convexity meningiomas, while incomplete removal of the tumors was observed mostly in cases of skull base meningiomas. The only risk factor for perioperative neurological deterioration was a critical tumor location such as localization at the skull base or in an eloquent area, as well as large vessels involvement by tumor. A worse physical status according to the ASA classification had an impact on overall morbidity, including the occurrence of neurological deterioration and/ or important, life-threatening internal complications. Due to a significantly higher risk of death, careful consideration should be taken for surgery in patients in a poor functional condition, ie with KPS $\leq 60$ and/or in a poor physical condition, ie with ASA 3 status. Hence, it seems that such patients should not be operated on and stereotactic radiosurgery or watchful observation is justified in these cases. The histopathological WHO grade of meningiomas predicts the recurrence rate in the elderly population and should be considered as an indicator of the need for postoperative radiotherapy. Irradiation is justified in cases of atypical meningiomas and noncomplete resection according to the Simpson classification (Simpson grades III or IV), and it should be obligatory in cases of anaplastic meningiomas. In the majority of patients who successfully survived the postoperative period, an improvement or at least a nonworsening of neurological status in relation to the preoperative condition was observed during long-term follow-up.

\section{Abbreviations}

ASA, American Society of Anesthesiologists; CART, classification and regression tree; CCS, Charlson Comorbidity Score; CT, computed tomography; CRGS, ClinicalRadiological Grading System; GKS, gamma-knife surgery; GSS, Geriatric Scoring System; IDH, isocitrate dehydrogenase; KPS, Karnofsky Performance Status; MRI, magnetic resonance imaging; MGMT, O-6-methylguanine-DNA methyltransferase; SRS, stereotactic radiosurgery; WHO, World Health Organization.

\section{Disclosure}

The author reports no conflicts of interest in this work.

\section{References}

1. Grossman R, Mukherjee D, Chang DC, et al. Preoperative charlson comorbidity score predicts postoperative outcomes among older intracranial meningioma patients. World Neurosurg. 2011;75 (2):279-285.

2. Modha A, Gutin P. Diagnosis and treatment of atypical and anaplastic meningiomas: a review. Neurosurgery. 2005;57(3):538-550. doi:10.1227/01.NEU.0000170980.47582.A5

3. Kuratsu J, Ushio Y. Epidemiological study of primary intracranial tumours in elderly people. J Neurol Neurosurg Psychiatry. 1997;63 (1):116-118. doi:10.1136/jnnp.63.1.116

4. Poon MT, Fung LH, Pu JK, Leung GK. Outcome of elderly patients undergoing intracranial meningioma resection-a systematic review and meta-analysis. $B r \quad J$ Neurosurg. 2014;28(3):303-309. doi:10.3109/02688697.2013.841857

5. Cohen-Inbar O, Soustiel J, Zaaroor M. Meningiomas in the elderly, the surgical benefit and a new scoring system. Acta Neurochir (Wien). 2010;152(1):87-97. doi:10.1007/s00701-009-0552-6

6. Patil C, Veeravagu A, Lad S, Boakye M. Craniotomy for resection of meningioma in the elderly: a multicentre, prospective analysis from the national surgical quality improvement program. $J$ Neurol Neurosurg Psychiatry. 2010;81(5):502-505. doi:10.1136/jnnp. 2009.185074

7. Bateman BT, Pile-Spellman J, Gutin PH, Berman MF. Meningioma resection in the elderly: nationwide inpatient sample, 1998-2002. Neurosurgery. 2005;57(5):866-872. doi:10.1227/01.NEU.000017 9923.66729.87

8. Karnofsky DA, Burchenal JH. The clinical evaluation of chemotherapeutic agents in cancer. In: MacLeod CM, editor. Evaluation of Chemotherapeutic Agents. New York: Columbia University Press; 1949:191-205.

9. Simpson D. The recurrence of intracranial meningiomas after surgical treatment. $J$ Neurol Neurosurg Psychiatry. 1957;20(1):22-39. doi:10.1136/jnnp.20.1.22

10. Caroli M, Locatelli M, Prada F, et al. Surgery for intracranial meningiomas in the elderly: a clinical-radiological grading system as a predictor of outcome. $J$ Neurosurg. 2005;102(2):290-294. doi:10.3171/jns.2005.102.2.0290 
11. Sacko O, Sesay M, Roux FE, et al. Intracranial meningioma surgery in the ninth decade of life. Neurosurgery. 2007;61(5):950-955. doi:10.1227/01.neu.0000303190.80049.7d

12. Bir S, Konar S, Maiti T, Guthikonda B, Nanda A. Surgical outcomes and predictors of recurrence in elderly patients with meningiomas. World Neurosurg. 2016;90:251-261. doi:10.1016/j.wneu.2016.02.066

13. Boviatsis E, Bouras T, Kouyialis A, Themistocleous M, Sakas D. Impact of age on complications and outcome in meningioma surgery. Surg Neurol. 2007;68(4):407-411. doi:10.1016/j.surneu.2006.11.071

14. D’Andrea G, Roperto R, Caroli E, Crispo F, Ferrante L. Thirty-seven cases of intracranial meningiomas in the ninth decade of life: our experience and review of the literature. Neurosurgery. 2005;56(5):956-961; discussion 956-961.

15. Dobran M, Marini A, Nasi D, et al. Surgical treatment and outcome in patients over 80 years old with intracranial meningioma. Clin Neurol Neurosurg. 2018;167:173-176. doi:10.1016/j. clineuro.2018.02.024

16. Roser F, Ebner F, Ritz R, Samii M, Tatagiba M, Nakamura M. Management of skull based meningiomas in the elderly patient. $J \quad$ Clin Neurosci. 2007;14(3):224-228. doi:10.1016/j. jocn.2005.12.004

17. Slot K, Peters J, Vandertop W, Verbaan D, Peerdeman S. Meningioma surgery in younger and older adults: patient profile and surgical outcomes. Eur Geriatr Med. 2018;9(1):95-101. doi:10.1007/ s41999-017-0015-1

18. Winther T, Torp S. Significance of the extent of resection in modern neurosurgical practice of World Health Organization grade I meningiomas. World Neurosurg. 2017;99:104-110. doi:10.1016/j. wneu.2016.11.034

19. Konglund A, Rogne SG, Helseth E, Meling T. Meningioma surgery in the very old-validating prognostic scoring systems. Acta Neurochir (Wien). 2013;155(12):2263-2271; discussion 2271. doi:10.1007/ s00701-013-1872-0

20. Yamamoto J, Takahashi M, Idei M, et al. Clinical features and surgical management of intracranial meningiomas in the elderly. Oncol Lett. 2017;14(1):909-917. doi:10.3892/ol.2017.6174

21. Hasseleid B, Meling TR, Rønning P, Scheie D, Helseth E. Surgery for convexity meningioma: simpson grade I resection as the goal. J Neurosurg. 2012;117(6):999-1006. doi:10.3171/2012.9.JNS12294

22. Poon MT, Fung LH, Pu JK, Leung GK. Outcome comparison between younger and older patients undergoing intracranial meningioma resections. J Neurooncol. 2013;114(2):219-227. doi:10.1007/ s11060-013-1173-8

23. Talacchi A, Muggiolu F, De Carlo A, Nicolato A, Locatelli F, Meglio M. Recurrent atypical meningiomas: combining surgery and radiosurgery in one effective multimodal treatment. World Neurosurg. 2016;87:565-572. doi:10.1016/j.wneu.2015.10.013

24. Lee K, DePowell J, Air E, Dwivedi A, Kendler A, McPherson C. Atypical meningiomas: is postoperative radiotherapy indicated? Neurosurg Focus. 2013;35(6):E15. doi:10.3171/2013.9. FOCUS 13325

25. Pollock B, Stafford S, Link M. Stereotactic radiosurgery of intracranial meningiomas. Neurosurg Clin N Am. 2013;24(4):499-507. doi:10.1016/j.nec.2013.05.006

26. Delgado-Fernández J, García-Pallero $\mathrm{M}$, Gil-Simoes $\mathrm{R}$, et al. Validation of grading scores and outcome prognostic factors in intracranial meningiomas in elderly patients. World Neurosurg. 2018;114: e1057-e1065. doi:10.1016/j.wneu.2018.03.146
27. Schul D, Wolf S, Krammer M, Landscheidt J, Tomasino A, Lumenta C. Meningioma surgery in the elderly: outcome and validation of 2 proposed grading score systems. Neurosurgery. 2012;70 (3):555-565. doi:10.1227/NEU.0b013e318233a99a

28. Cohen-Inbar O, Lee C, Schlesinger D, Xu Z, Sheehan J. Long-term results of stereotactic radiosurgery for skull base meningiomas. Neurosurgery. 2016;79(1):58-68. doi:10.1227/NEU.0000000000001045

29. Kaul D, Budach V, Graaf L, Gollrad J, Badakhshi H. Outcome of elderly patients with meningioma after image-guided stereotactic radiotherapy: a study of 100 cases. Biomed Res Int. 2015;Article ID:868401.

30. Nakamura M, Roser F, Michel J, Jacobs C, Samii M. The natural history of incidental meningiomas. Neurosurgery. 2003;53(1):62-70; discussion 70-71. doi:10.1227/01.NEU.0000068730.76856.58

31. Yoneoka Y, Fujii Y, Tanaka R. Growth of incidental meningiomas. Acta Neurochir (Wien). 2000;142(5):507-511. doi:10.1007/ s007010050463

32. Nakasu S, Fukami T, Nakajima M, Watanabe K, Ichikawa M, Matsuda M. Growth pattern changes of meningiomas: long-term analysis. Neurosurgery. 2005;56(5):946-954.

33. Klinger D, Flores B, Lewis J, et al. Atypical meningiomas: recurrence, reoperation, and radiotherapy. World Neurosurg. 2015;84 (3):839-845. doi:10.1016/j.wneu.2015.04.033

34. Mair R, Morris K, Scott I, Carroll T. Radiotherapy for atypical meningiomas. J Neurosurg. 2011;115(4):811-819. doi:10.3171/ 2011.5.JNS11112

35. Pearson B, Markert J, Fisher W, et al. Hitting a moving target: evolution of a treatment paradigm for atypical meningiomas amid changing diagnostic criteria. Neurosurg Focus. 2008;24(5):E3. doi:10.3171/FOC/2008/24/5/E3

36. Ius $\mathrm{T}$, Somma T, Altieri R, et al. Is age an additional factor in the treatment of elderly patients with glioblastoma? A new stratification model: an Italian Multicenter Study. Neurosurg Focus. 2020;49(4): E13. doi:10.3171/2020.7.FOCUS20420

37. Wang Y, Zhao B, Chen W, et al. Pretreatment geriatric assessments of elderly patients with glioma: development and implications. Aging Dis. 2020;11(2):448-461. doi:10.14336/AD.2019.0527

38. Young J, Chmura S, Wainwright D, Yamini B, Peters K, Lukas R. Management of glioblastoma in elderly patients. J Neurol Sci. 2017;380:250-255. doi:10.1016/j.jns.2017.07.048

39. Bowers C, Gurgel R, Brimley C, et al. Surgical treatment of vestibular schwannoma: does age matter? World Neurosurg. 2016;96:58-65. doi:10.1016/j.wneu.2016.08.054

40. Tardivo V, Penner F, Garbossa D, et al. Surgical management of pituitary adenomas: does age matter? Pituitary. 2020;23(2):92-102. doi:10.1007/s11102-019-01014-1

41. Zhan R, Ma Z, Wang D, Li X. Pure endoscopic endonasal transsphenoidal approach for nonfunctioning pituitary adenomas in the elderly: surgical outcomes and complications in 158 patients. World Neurosurg. 2015;84(6):1572-1578. doi:10.1016/j.wneu.2015.08.035

42. Zhao Y, Lian W, Xing B, et al. The clinical characteristics and microsurgical therapy of pituitary adenomas in elderly patients: a retrospective study of 130 cases. J Clin Neurosci. 2017;46:13-16. doi:10.1016/j.jocn.2017.08.038

43. Sylvester M, Shastri D, Patel V, et al. Outcomes of vestibular schwannoma surgery among the elderly: analysis of the national inpatient sample. Otolaryngol Head Neck Surg. 2017;156 (1):166-172. doi:10.1177/0194599816677522 


\section{Publish your work in this journal}

Clinical Interventions in Aging is an international, peer-reviewed journal focusing on evidence-based reports on the value or lack thereof of treatments intended to prevent or delay the onset of maladaptive correlates of aging in human beings. This journal is indexed on PubMed Central, MedLine, CAS, Scopus and the Elsevie
Bibliographic databases. The manuscript management system is completely online and includes a very quick and fair peer-review system, which is all easy to use. Visit http://www.dovepress.com/ testimonials.php to read real quotes from published authors. 